QMW-PH-97-41

\title{
Logarithmic Conformal Field Theory Solutions of Two Dimensional Magnetohydrodynamics
}

\author{
Spyros Skoulakis 7 and Steven Thomast \\ Department of Physics \\ Queen Mary and Westfield College \\ Mile End Road \\ London E1 \\ U.K.
}

\begin{abstract}
We consider the application of logarithmic conformal field theory in finding solutions to the turbulent phases of 2-dimensional models of magnetohydrodynamics. These arise upon dimensional reduction of standard (infinite conductivity) 3-dimensional magnetohydrodynamics, after taking various simplifying limits. We show that solutions of the corresponding Hopf equations and higher order integrals of motion can be found within the solutions of ordinary turbulence proposed by Flohr, based on the tensor product of the logarithmic extension $\tilde{c}_{6,1}$ of the non-unitary minimal model $c_{6,1}$. This possibility arises because of the existence of a continuous hidden symmetry present in the latter models, and the fact that there appear several distinct dimension -1 and -2 primary fields.
\end{abstract}

\footnotetext{
${ }^{1}$ email: s.skoulakis@qmw.ac.uk

${ }^{2}$ email: s.thomas@qmw.ac.uk
} 


\section{Introduction to Polyakov turbulence and Flohr solution}

The word turbulence is used to describe the irregular and fluctuating motion that is seen in many cases of gas and liquid flow. We can have a qualitative description of the flow by a dimensionless number, the Reynolds number $R e$, which measures the relative strength of inertial and viscous forces acting on the fluid. Turbulence is associated with flows having very high Reynolds number. Formally we can take $\operatorname{Re} \rightarrow \infty$ and this is the case of the fully developed turbulent flow. The Navier-Stokes equation describes these flows in 2 and 3 dimensions. One of the main differences between inviscid flows in each of these dimensions, is the existence of an infinite number of conserved quantities in 2-dimensions, and it is these and their corresponding conserved fluxes that make two dimensional turbulence particularly interesting. Indeed there has been a growing body of research on the subject since the classic work of Kraichnan [1].

A fresh approach to the problem of understanding turbulent flows in $d=2$ was presented by Polyakov $[2,3]$, who, motivated by the success of Conformal Field Theory (CFT) in describing 2D critical phenomena, suggested that the methods of CFT can help in this task. The central point in the analysis was the Hopf equation, written here in terms of the vorticity $\omega=\epsilon_{\alpha \beta} \partial_{\alpha} v_{\beta}, \quad \alpha, \beta=1,2$

$$
\sum_{p=1}^{n}\left\langle\omega\left(x_{1}\right) \cdots \partial_{t} \omega\left(x_{p}\right) \cdots \omega\left(x_{n}\right)\right\rangle=0
$$

where the time derivative of $\omega$ appearing in eq(11) is given in terms of the Navier -Stokes equations

$$
\partial_{t} \omega+\epsilon_{\alpha \beta} \partial_{\alpha} \psi \partial_{\beta} \omega=\nu \partial^{\alpha} \partial_{\alpha} \omega
$$

where, assuming incompressibility, the velocity is expressed in terms of the stream function $\psi$ as $v_{\alpha}=\epsilon_{\alpha \beta} \partial_{\beta} \psi$

The Hopf equations express $n$-point function in terms of the $n+1$ point functions. They can't be solved directly and the only way so far was to take ad-hoc approximations and try to find solutions. In contrast, with the Conformal approach we try to solve the Hopf equation exactly by the ansatz that the flow has conformal symmetry in the situation where it is fully developed.

The assumption is that there exists a range of scales such that the affect of the viscocity term is very small - the so called inertial range. So we insert $\omega=-\partial^{2} \psi$ in 
the Hopf equation, postulating that $\psi$ is a primary operator of the CFT we are looking for. Because of this, one is naturally lead to introduce point splitting regularization in order to evaluate products of fields defined at the same point. Using the operator product expansion (OPE) for $\psi$ with itself

$$
[\psi] \times[\psi]=[\phi]+\ldots
$$

where $\phi$ is the conformal field with the minimal dimension appearing in the OPE, and $+\ldots$ indicates next to leading order terms, the Navier-Stokes equations imply that

$$
\dot{\omega}=-|a|^{2\left(\Delta_{\phi}-2 \Delta_{\psi}\right)} \mathcal{L} \phi, \quad \mathcal{L}=\left[L_{-2} \bar{L}_{-1}^{2}-\bar{L}_{-2} L_{-1}^{2}\right]
$$

appears inside the correlators in the Hopf equation, where $a$ is the u.v. cutoff associated with point splitting. In principle, a solution to the Hopf equations can be found if the operator on the right hand side in eq(田) is vanishing. However, this requirement by itself is not restrictive enough to allow us to find explicit solutions. To place further restrictions, one has to take into account the spectrum associated with turbulence. One of the predictions of the conformal approach is that the $k$-space kinetic energy density $E(k)$ (defined by $\int d k E(k)=\left\langle v_{\alpha}(k) v_{\alpha}(-k)\right\rangle$ ) implies a power law spectrum with $E \sim k^{4 \Delta_{\psi}+1}$. In the classical turbulence studies, the value of the exponent of $k$ in the above relation was one of the first things to be studied. There are different opinions for its value but the work of Kraichnan [1] indicated that in the case of the inverse cascade scenario, (similar to the Kolmogorov one in $d=3$ ), $E \sim k^{-3}$ which is based on the constancy of the enstropy flux. So it is natural to postulate this cascade scenario in order to increase our restrictions on the possible conformal field theory solutions. In this case we have

$$
\langle\dot{\omega}(z, \bar{z}) \omega(0)\rangle \sim \text { const } \Rightarrow \Delta_{\phi}+\Delta_{\psi}+3=0
$$

Trying to find solutions of these constraints, Polyakov considered the possibility $2 \Delta_{\psi}<\Delta_{\phi}$, which 'trivially' satisfies the Hopf equations in $|a| \rightarrow 0$ limit, and found solutions within the non-unitary minimal models. In fact hundreds of solutions can be found in this way [4-9], which is perhaps an indication that this approach is not encapsulating all the physical aspects of turbulence we would like. Worse still, Falkovich and Hanany [10], showed that a solution that has the Kraichnan spectrum can not be found within the minimal models. Because of the constancy of all the higher order 
fluxes, they found that $2 \Delta_{\psi}=\Delta_{\phi}=-2$ and this case is not found in the minimal models. Also in the minimal model case the actual prediction is that all fluxes vanish so the situation is made more difficult. Such difficulties indicate perhaps that the 'usual' Conformal approach might describe certain kinds of flows, but not the turbulent ones.

In an attempt to directly deal with these issues, Flohr [11] has suggested a solution that does not suffer from these problems and is based on Logarithmic Conformal Theories (LCFT) [12 -16]. This ability to avoid the aforementioned difficulties and yet still remain within the conformal approach is clearly due to the novel characteristics of LCFT's.

One such important characteristic, is the existence of pairs of primary fields $C(x), D(x)$ in the spectrum having the same dimension and with respect to which the Virasoro operator $L_{0}$ is non-diagonal i.e. $L_{0}|C\rangle=\Delta_{C}|C\rangle, \quad L_{0}|D\rangle=\Delta_{D}|D\rangle+|C\rangle$.

The two point functions of the logarithmic pairs $C(x), D(x)$ have the following form (see e.g. [13])

$$
\begin{aligned}
\langle C(x) D(y)\rangle & =\frac{d}{(x-y)^{2 \Delta_{C}}} \\
\langle D(x) D(y)\rangle & =\frac{d}{(x-y)^{2 \Delta_{C}}}(-2 e \ln (x-y)+d) \\
\langle D(x) D(y)\rangle & =0
\end{aligned}
$$

where $d, e$ are constants. As pointed out in [13], the first correlation in eq(6) together with the invariance of the correlator under exchange of $C(x)$ and $D(x)$ implies a strong constraint on the allowed values of the dimensions $\Delta_{C}$, namely that it be integer.

This implies that the logarithmic operators $C(x)$ (or rather their chiral components) are associated with a hidden continuous symmetry, although unlike the more usual current algebras, the Schwinger term is vanishing.

This hidden continuous symmetry associated with LCFT's is an important ingredient in Flohr's solution of the Hopf equations (1).

In searching for possible non-unitary LCFT solutions to turbulence, (the nonunitarity is a consequence of the flux constraint (5)), the logarithmic extensions of the minimal model CFT's is perhaps the first place to start since a certain amount

\footnotetext{
${ }^{3}$ See also [26] for additional clarification of this point
} 
of knowledge already exists about their properties [16]. In fact it is the $\tilde{c}_{p, 1}$ models that have been studied most. As we have seen above, the Kraichnan spectrum implies that there should be $\Delta=-1$ and -2 states in the spectrum of our LCFT. This is not possible in a single copy of $\tilde{c}_{p, 1}$ but as shown by Flohr [11], since $\tilde{c}_{6,1}$ does contain a $\Delta=-1$ field, the tensor product $\tilde{c}_{6,1} \otimes \tilde{c}_{6,1}$ can contain both. The additional advantage of the tensor product model is that it naturally contains Polyakov's Bose condensate [3] within the solution.

$\tilde{c}_{6,1}$ has 17 primary fields, 5 of them are "logarithmic". The dimensions of the fields are $\Delta_{1, r}=\frac{1}{4 p}\left((p-r)^{2}-(p-1)^{2}\right)$ with $p=6$.

Because of the tensor product there is a degeneracy in the way we can define the states with dimension $-1,-2$. In fact this will prove to be a nice feature of the solution, which we shall exploit later on when considering the MHD case.

We define the $\Delta=-1$ and $\Delta=-2$ fields, $\psi_{i}, \phi_{j^{\prime}}$ as follows (with $\Phi_{\left(\Delta \mid \Delta^{\prime}\right)} \equiv$ $\left(\Phi_{\Delta} \otimes \Phi_{\Delta^{\prime}}\right)(z)$, and $\Phi_{\Delta}(z)$ a primary field in $\left.\tilde{c}_{6,1}\right)$

$$
\begin{aligned}
& \psi_{1}=\frac{1}{2}\left(\Phi_{(-1 \mid-\tilde{0})}+\Phi_{(-\tilde{0} \mid-1)}\right), \quad \psi_{2}=\frac{1}{2}\left(\Phi_{(-\tilde{1} \mid-\tilde{0})}+\Phi_{(-\tilde{0} \mid-\tilde{1})}\right) \\
& \phi_{1}=\Phi_{(-1 \mid-1)}, \quad \phi_{2}=\frac{1}{2}\left(\Phi_{(-1 \mid-\tilde{1})}+\Phi_{(-\tilde{1} \mid-1)}\right), \quad \phi_{3}=\Phi_{(-\tilde{1} \mid-\tilde{1})}
\end{aligned}
$$

It is easy to see that $\psi_{i} \times \psi_{j}=\sum_{k^{\prime}} C_{i j}^{k^{\prime}} \phi_{k^{\prime}}+\ldots, i, j=1,2$ and $k^{\prime}=1,2,3$, where the OPE coefficients $C_{i j k^{\prime}}$ can be obtained from the fusion rules of $\tilde{c}_{6,1}$ LCFT given in [16].

We expect the physical fields to be given by linear combinations of the fields $\psi_{i}$ and $\phi_{j^{\prime}}$, i.e. $\psi_{p h}=\sum_{i} \alpha_{i} \psi_{i}$ and $\phi_{p h}=\sum_{j^{\prime}} \beta_{j^{\prime}} \phi_{j^{\prime}}$ with

$$
\psi_{p h} \times \psi_{p h}=\phi_{p h}+\ldots
$$

In order that the physical fields $\psi_{p h}$ satisfy eq(8), the coefficients $\alpha_{i}$ and $\beta_{j^{\prime}}$ must satisfy $\sum_{i j} \alpha_{i} \alpha_{j} C_{i j k^{\prime}}=\beta_{k^{\prime}}$ which essentially determines the coefficients $\beta_{k^{\prime}}$ once the $\alpha_{i}$ are known.

From the general form of $\psi_{p h}$ one can determine that its $k$-space two-point function has the structure

$$
\left\langle\psi_{p h}(k) \psi_{p h}(-k)\right\rangle=\frac{a_{0}}{|k|^{2-4 \Delta_{\psi}}}+\frac{a_{1}}{L^{2 \Delta_{\psi}}} \delta(k)+\ldots
$$

with the coefficients $a_{0} \sim\langle I\rangle$ and $a_{1} \sim\left\langle\phi_{p h}\right\rangle$. A notable feature as pointed out in [11], is the presence of the so called Polyakov condensate term (proportional to $\delta(k)$ ) which is naturally contained within the solution. 
Flohr considered a more general expansion of the physical fields $\psi_{p h}$ and $\phi_{p h}$ involving non-diagonal combinations of left and right-moving states. This leads to non-zero spin contributions to $\psi_{p h}$ and $\phi_{p h}$ that may naturally encode the effect of stirring forces in the fluid. The general expansions are of the form

$$
\begin{aligned}
& \hat{\psi}_{p h}(z, \bar{z})=\psi_{p h}(z) \times \psi_{p h}(\bar{z})+\sum_{\Delta_{U}+\bar{\Delta}_{V}=-2, \Delta_{U}+\bar{\Delta}_{V} \in Z} U(z) \times V(\bar{z}) \\
& \hat{\phi}_{p h}(z, \bar{z})=\phi_{p h}(z) \times \phi_{p h}(\bar{z})+\sum_{\Delta_{U^{\prime}}+\bar{\Delta}_{V^{\prime}}=-4, \Delta_{U^{\prime}}+\bar{\Delta}_{V^{\prime}} \in Z} U^{\prime}(z) \times V^{\prime}(\bar{z})
\end{aligned}
$$

where the integer dimension fields $U(z), V(\bar{z}), U^{\prime}(z), V^{\prime}(\bar{z})$ are those occuring in $\tilde{c}_{6,1} \otimes$ $\tilde{c}_{6,1}$. This leads to the following expression for $\hat{\psi}_{p h}$

$$
\begin{aligned}
\hat{\psi}_{p h}(z, \bar{z}) & =a_{1} \Phi_{(-1 \mid 0)}(z) \otimes \bar{\Phi}_{(-1 \mid 0)}(\bar{z})+a_{2} \Phi_{(0 \mid-1)}(z) \otimes \bar{\Phi}_{(0 \mid-1)}(\bar{z}) \\
& +a_{3} \Phi_{(-1 \mid \tilde{0})}(z) \otimes \bar{\Phi}_{(-1 \mid \tilde{0})}(\bar{z})+a_{4} \Phi_{(\tilde{0} \mid-1)}(z) \otimes \bar{\Phi}_{(\tilde{0} \mid-1)}(\bar{z}) \\
& +a_{5} \Phi_{(-1 \mid-1)}(z) \otimes \bar{\Phi}_{(0 \mid 0)}(\bar{z})+a_{6} \Phi_{(0 \mid 0)}(z) \otimes \bar{\Phi}_{(-1 \mid-1)}(\bar{z}) \\
& +a_{7} \Phi_{(0 \mid 0)}(z) \otimes \bar{\Phi}_{(\tilde{0} \mid \tilde{0})(\bar{z})}+\cdots
\end{aligned}
$$

There is a similar expansion of $\hat{\phi}_{p h}(z, \bar{z})$. The non-diagonal terms in eq(11) induce further terms in the two-point function eq(9) that are proportional to the expectation values of chiral fields, and involve the derivative of $k$-space delata functions [11]. A crucial aspect of the model is the existance of a hidden continuous symmetry [13] associated with the integer dimension field $\Psi_{\Delta_{1,5}}$. In fact this guarantees that it is a solution to the Hopf equations. The operator $\Omega(z)=L_{0} \bar{L}_{0}\left[L_{-2} \bar{L}_{-1}^{2}-\bar{L}_{-2} L_{-1}^{2}\right] \phi_{p h}(z)$ generates a continuous symmetry of the LCFT and satisfies the condition f

$$
\left\langle\Omega(z) \Phi_{1}\left(z_{1}\right) \Phi_{2}\left(z_{2}\right) \ldots \Phi_{n}\left(z_{n}\right)\right\rangle=0
$$

In ending this section, it is perhaps worth mentioning the reason one finds nonunitary CFT in connection with turbulence, may in some sense be expected, since the turbulent state is not an equilibrium state. Even if the conformal solutions don't

\footnotetext{
${ }^{4}$ As argued in [11], The presence of $L_{0} \bar{L}_{0}$ in $\Omega(z)$ is needed to ensure that only the $\Psi_{\Delta_{1,5}}$ part of $\phi_{p h}$ enters the Hopf equations. This follows from certain properties of correlation functions of LCFT discussed in [15]
} 
describe the turbulent state exactly, we might at least expect them to describe flows out of equilibrium.

Motivated by the new possibilities that LCFT has brought to conformal turbulence, in the next section we want to extend Flohr's construction and look for solutions of the generalized turbulent MHD systems in $d=2$ that arise naturally when dimensionally reducing standard ideal MHD in $d=3$.

\section{Logarithmic solutions to MHD turbulence}

The long wavelength limit of 3-dimensional plasmas are well approximated by the equations of Magnetohydrodynamics (MHD), which in the limit of low frequencies, low temperature and incompressibility take the simplified form [17]

$$
\begin{aligned}
\nabla \cdot \mathbf{v} & =0 \\
\nabla \cdot \mathbf{B} & =0 \\
\partial_{t} \mathbf{v}+\mathbf{v} \cdot \nabla \mathbf{v} & =-\frac{1}{\rho_{m}} \nabla P+\frac{1}{c \rho_{m}} \mathbf{J} \times \mathbf{B} \\
\nabla \times(\mathbf{v} \times \mathbf{B}) & =\partial_{t} \mathbf{B} \\
\nabla \times \mathbf{B} & =\frac{4 \pi}{c} \mathbf{J}
\end{aligned}
$$

Here $\mathbf{v}, \mathbf{B}, \mathbf{J}$ are the velocity field, magnetic field and electric current respectively. $P$ and $\rho_{m}$ are the pressure and mass density. After simple manipulations, we can recast these equations into a more symmetric form

$$
\begin{aligned}
\nabla \cdot \mathbf{v} & =0 \\
\nabla \cdot \mathbf{B} & =0 \\
\nabla \times\left(\partial_{t} \mathbf{v}+\mathbf{v} \cdot \nabla \mathbf{v}\right) & =\frac{1}{4 \pi \rho_{m}} \nabla \times(\mathbf{B} \cdot \nabla \mathbf{B}) \\
(\mathbf{B} \cdot \nabla) \mathbf{v}-(\mathbf{v} \cdot \nabla) \mathbf{B} & =\partial_{t} \mathbf{B}
\end{aligned}
$$

In order to obtain an effectively two-dimensional theory from eq(14), one simple proposal is to dimensionally reduce the model by requiring that all fields be independent of the $z$-coordinate, i.e. $\partial_{3} \mathbf{v}=0$ and $\partial_{3} \mathbf{B}=0$. It follows that the first two components of $\mathbf{v}$ and $\mathbf{B}$ can be written as

$$
B_{\alpha}=\epsilon_{\alpha \beta} \partial_{\beta} A, \quad v_{\alpha}=\epsilon_{\alpha \beta} \partial_{\beta} \psi, \quad \alpha, \beta=1,2
$$


One can define, in analogy with vorticity $\omega$, the "magnetic vorticity"

$$
\Omega \equiv \epsilon_{\alpha \beta} \partial_{\alpha} B_{\beta}=-\partial_{\alpha} \partial_{\alpha} A,
$$

and the two-dimensional operator

$$
\mathcal{A} \equiv \epsilon_{\alpha \beta} \partial_{\beta} A \partial_{\alpha}
$$

after which the 2-D MHD equations take the form [18]:

$$
\begin{aligned}
\dot{\omega}+\epsilon_{\alpha \beta} \partial_{\beta} \psi \partial_{\alpha} \omega & =\frac{1}{4 \pi \rho_{m}} \mathcal{A} \Omega \\
\dot{A}+\epsilon_{\alpha \beta} \partial_{\beta} \psi \partial_{\alpha} A & =0 \\
\dot{V}+\epsilon_{\alpha \beta} \partial_{\beta} \psi \partial_{\alpha} V & =\frac{1}{4 \pi \rho_{m}} \mathcal{A} B \\
\dot{B}+\epsilon_{\alpha \beta} \partial_{\beta} \psi \partial_{\alpha} B & =\mathcal{A} V
\end{aligned}
$$

Solutions to eqs(18) have been studied in the context of minimal model CFT, in two simplifying cases, namely parallel flow conditions where $B=V=0$ [18], and perpendicular flow for which $A=V=0$ [19]. In both cases two of the four equations in (18) are automatically satisfied and the remaining equations to be studied are:

$$
\begin{aligned}
\dot{\omega}+\epsilon_{\alpha \beta} \partial_{\beta} \psi \partial_{\alpha} \omega & =\frac{1}{4 \pi \rho_{m}} \mathcal{A} \Omega \\
\dot{A}+\epsilon_{\alpha \beta} \partial_{\beta} \psi \partial_{\alpha} A & =0
\end{aligned}
$$

for parallel flow and

$$
\begin{aligned}
& \dot{\omega}+\epsilon_{\alpha \beta} \partial_{\beta} \psi \partial_{\alpha} \omega=0 \\
& \dot{B}+\epsilon_{\alpha \beta} \partial_{\beta} \psi \partial_{\alpha} B=0
\end{aligned}
$$

for perpendicular flow.

We want to discuss the behaviour of various fluxes when one considers dissapative forces. In the case of ordinary fluids this means reintroducing the viscosity $\nu$. In the MHD case there is an additional dissapation parameter namely the conductivity $\sigma$. Keeping $\nu=0$ for the present but $\sigma$ finite, we find for the equations of motion of perpendicular flow [19] 


$$
\begin{aligned}
\dot{\omega}+\epsilon_{\alpha \beta} \partial_{\beta} \psi \partial_{\alpha} \omega & =-\frac{\rho_{c}}{\rho_{m}}\left(\frac{c}{4 \pi \sigma} \partial_{\alpha} \partial_{\alpha} B-\frac{1}{c}\left[\epsilon_{\alpha \beta} \partial_{\beta} \psi \partial_{\alpha} B\right]\right) \\
\dot{B}+\epsilon_{\alpha \beta} \partial_{\beta} \psi \partial_{\alpha} B & =\frac{c^{2}}{4 \pi \sigma} \partial_{\alpha} \partial_{\alpha} B
\end{aligned}
$$

Finite viscosity $\nu$ would add the standard dissapative term to the rhs of the $\omega$ equation of motion but leave that of $B$ unaffected. Hence viscosity and conductivity are responsible for the decay of higher point enstrophy and magnetic fluxes, $H_{n}(k, t)$ and $\mathcal{B}_{n}(k, t)$ where

$$
\int d k H_{n}(k, t)=\int d^{2} x\left\langle\omega^{n}(x)\right\rangle, \quad \int d k \mathcal{B}_{n}(k, t)=\int d^{2} x\left\langle B^{n}(x)\right\rangle
$$

For infinite conductivity but non-vanishing $\nu, \mathcal{B}_{n}$ is time independent (so $B$-fluxes are conserved) but $H_{n}$ satisfies

$$
\dot{H}_{n} \sim \nu \int_{L^{-1}}^{a^{-1}} k^{2} h_{n}(k, t) d k
$$

where we have introduced the I.R. cutoff $L$. Dimensional analysis implies that the viscosity $\nu$ and ultraviolet cutoff $a$ are related via $\nu \sim a^{2 \Delta_{\psi}-2 \Delta_{\phi}}$. A further analysis on the viscosity dependence of $H_{n}(k)$ implies [5]

$$
\dot{H}_{n} \sim \nu^{\left[(n-1)\left(\Delta_{\psi}+1\right)+\Delta_{\phi}+2\right] /\left(\Delta_{\phi}-\Delta_{\psi}\right)}
$$

Demanding that the higher order fluxes $H_{n}$ have the same scaling properties independent of $n$ determines the scaling dimensions of both $\phi$ and $\psi$ to be $\Delta_{\psi}=$ $-1, \Delta_{\phi}=-2$ and immediately gives rise to the Kraichnan form for the energy spectrum $E(k) \sim k^{-3}[5]$.

In the present case of MHD we can also imagine considering the situation where we have inviscid flow but with $\sigma$ finite. In this case we can perform a similar dimensional analysis as before. The relation between $\sigma$ and $a$ can be determined from the $B$ equation of motion (20) to be $\sigma^{-1} \sim a^{\left(2 \Delta_{B}-2 \Delta_{\chi^{\prime}}-2\right)}$, where $\chi^{\prime}$ is defined to be the minimal dimension field in the OPE $B \times \Psi$. The fluxes $H_{n}$ and $\mathcal{B}_{n}$ then satisfy

$$
\begin{aligned}
\dot{H}_{n} & \sim \frac{\rho_{e}}{\rho_{m}} \sigma^{-1} \int d^{2} x\left\langle\omega^{n-1} \partial^{2} B\right\rangle+\ldots \\
\dot{\mathcal{B}} & \sim \sigma^{-1} \int d^{2} x\left\langle B^{n-1} \partial^{2} B\right\rangle
\end{aligned}
$$


It follows that the operators $\dot{\omega}$ and $\dot{B}$ have the same dimension and hence $\Delta_{\chi^{\prime}}=$ $\Delta_{\phi}=-2$ for consistency. The $\sigma$ dependence of $\mathcal{B}_{n}$ can be obtained as

$$
\mathcal{B}_{n} \sim \sigma^{\left[(n-2) \Delta_{B}+\Delta_{\chi^{\prime}}+2\right] /\left(\Delta_{B}-\Delta_{\chi^{\prime}}\right)}
$$

Hence it follows that the scaling behaviour of $\mathcal{B}_{n}$ is independent of $n$ if $\Delta_{B}=0$ and $\Delta_{\chi^{\prime}}=-2$, and we see the condition on $\Delta_{\chi^{\prime}}$ agrees with the one found above by consistency.

In studying the possible solutions of the inviscid ideal MHD equations, in the perpendicular flow regime eq(20), we have to identify the dimension zero field $B$ with certain primary operators. We want to look for solutions of the resulting Hopf equations similar to the logarithmic conformal field theory solutions considered by Flohr in the case of normal fluids. An obvious motivation for this is that the LCFT $\tilde{c}_{6,1} \otimes \tilde{c}_{6,1}$ considered in [11] solves the Hopf equation involving $\omega$, which is one of the equations in (20). The hope is (and we shall see that this is indeed possible) that one can identify the $B$ field with primary fields within this particular LCFT in such a way as the Hopf equations associated with the second equation in (20) can be satisfied. Even though this is in the context of perpendicular flow, one should expect that similar ideas would apply to the parallel case as well.

Indeed, considering the equation of parallel flow, eq(19), one obtains after performing the regularization and OPE's,

$$
\begin{aligned}
\dot{\omega} & =|a|^{2\left(\Delta_{\varphi}-2 \Delta_{A}\right)} \mathcal{L} \varphi-|a|^{2\left(\Delta_{\phi}-2 \Delta_{\psi}\right)} \mathcal{L} \phi=0 \\
\dot{A} & =|a|^{2\left(\Delta_{\chi}-\Delta_{A}-\Delta_{\psi}\right)} \mathcal{L} \chi=0
\end{aligned}
$$

where in eq(27), $\mathcal{L}=\left[L_{-2} \bar{L}_{-1}^{2}-\bar{L}_{-2} L_{-1}^{2}\right]$ and $A \times A=[\varphi]+\cdots, \psi \times \psi=[\phi]+\cdots$ and $A \times \psi=[\chi]+\cdots$. Here $\varphi, \phi$ and $\chi$ are minimal dimension fields.

Perpendicular flow corresponds to the equations

$$
\begin{aligned}
& \dot{\omega}=-|a|^{2\left(\Delta_{\phi}-2 \Delta_{\psi}\right)} \mathcal{L} \phi=0 \\
& \dot{B}=-|a|^{2\left(\Delta_{\chi^{\prime}}-\Delta_{\psi}-\Delta_{B}\right)} \mathcal{L} \chi^{\prime}=0
\end{aligned}
$$

where $\chi^{\prime}$ is the minimal dimension field in the OPE $B \times \psi=\left[\chi^{\prime}\right]+\cdots$.

Now we have seen previously that flux conditions dictate that $\Delta_{B}=0$. Also one finds that on dimensional grounds $\Delta_{A}=0$. Thus both these fields have to be 
constructed from $\Delta=0$ fields in the $\tilde{c}_{6,1} \times \tilde{c}_{6,1}$ LCFT. Just as in the case of the $\Delta=-1,-2$ states, there are several possible primary fields of dimension zero, which we call $b_{i^{\prime}}, i^{\prime}=1,2,3$, where

$$
\begin{aligned}
& b_{1}=\Phi_{(\tilde{0} \mid \tilde{0})}, \quad b_{2}=\frac{1}{2}\left(\Phi_{(1 \mid-1)}+\Phi_{(-1 \mid 1)}\right) \\
& b_{3}=\frac{1}{2}\left(\Phi_{(1 \mid-\tilde{1})}+\Phi_{(-\tilde{1} \mid 1)}\right)
\end{aligned}
$$

(There are other possibilities involving tensor products containing the identity operator $I$ which we do not include as they are in some sense trivial).

As in the case of ordinary turbulence, we expect that the physical components of the magnetic field $B$ and $A$ will be given by some linear combination of the $b_{i}$ fields. Under these circumstances, in verifying either of the two sets of Hopf equations following from eqs (27, 28) we need to calculate the OPE's $b_{i^{\prime}} \times b_{j^{\prime}}$ and $b_{i^{\prime}} \times \psi_{j}$. In order to obtain these expansions we need some further fusion rules of $\tilde{c}_{6,1}$ beyond those considered in [11]. Specifically we need those involving the primary field $[1]$ with the set $[\tilde{0}],[-1],[-\tilde{1}],[1]$, which can be obtained from the general rules presented in reference [15],

$$
\begin{aligned}
{[1] \times[\tilde{0}] } & =\left[-\frac{2}{3}\right]+[-1]+[-\tilde{1}]+\left[-\frac{\tilde{2}}{3}\right]+[\tilde{0}]+[1]+\left[\frac{7}{3}\right]+[4] \\
{[1] \times[-1] } & =\left[-\frac{2}{3}\right]+[\tilde{0}]+[1]+\left[\frac{7}{3}\right]+[4] \\
{[1] \times[-\tilde{1}] } & =[-\tilde{1}]+\left[-\frac{\tilde{2}}{3}\right]+[\tilde{0}]+[1]+\left[\frac{7}{3}\right]+[4] \\
{[1] \times[1] } & =[\tilde{0}]+\left[-\frac{2}{3}\right]+[-1]+\left[-\frac{\tilde{2}}{3}\right]+[1]+\left[\frac{7}{3}\right]+[4]
\end{aligned}
$$

where the right hand side of the OPE's in eq(30) is to be understood modulo multiplicities and subject to the linear relation amongst certain conformal families as explained in detail in [16]. Using these relations and those defined earlier in section 1, one can obtain

$$
\begin{aligned}
b_{1} \times \psi_{1} & =4 \phi_{2} \\
b_{1} \times \psi_{2} & =2 \phi_{1} \\
b_{2} \times \psi_{1} & =\frac{1}{2} \phi_{1}+\phi_{2}+\frac{1}{2} \phi_{3} \\
b_{2} \times \psi_{2} & =\phi_{2}+\phi_{1}
\end{aligned}
$$




$$
\begin{aligned}
b_{3} \times \psi_{1} & =\frac{1}{2} \phi_{1}+\frac{1}{2} \phi_{2} \\
b_{3} \times \psi_{2} & =\phi_{2}+\frac{1}{2} \phi_{1} \\
b_{1} \times b_{1} & =\phi_{1} \\
b_{1} \times b_{2} & =\frac{1}{2} \phi_{1}+\phi_{2}+\frac{1}{2} \phi_{3} \\
b_{1} \times b_{3} & =\phi_{1}+\phi_{2} \\
b_{2} \times b_{3} & =\frac{1}{4} \phi_{1}+\frac{1}{2} \phi_{2} \\
b_{3} \times b_{3} & =\frac{1}{2} \phi_{1}+\frac{1}{2} \phi_{3}+\frac{1}{2} \phi_{2}
\end{aligned}
$$

An important feature of the OPE in eq(31) is that the leading terms on the right hand side (i.e. those with smallest dimensions) are those of $[-1]$ and $[-\tilde{1}]$, except in the OPE [1] $\times[-1]$ where the lowest dimension is $-2 / 3$. Despite this exception one finds that the leading order representations on the rhs of eq(30) are just given by linear combinations of the $\phi_{i}$ fields defined earlier.

In the case of parallel flow the solution includes a field with dimension $-1(\psi)$, one field with dimension $0(A)$ and 3 fields with dimension $-2,(\phi, \chi, \varphi)$. In the case of perpendicular flow we have again $\psi, B$ with dimension 0 and $\chi^{\prime}$ with dimension -2 . We see that the solution accommodates both cases because of the freedom we have in the definition of the physical fields. We define the physical fields $\phi_{p h}, \psi_{p h}$ as in the previous section (for simplicity we do not consider non-diagonal combinations at this point), and $A_{p h}=\sum_{i^{\prime}} \gamma_{i^{\prime}} b_{i^{\prime}}, \quad B_{p h}=\sum_{i^{\prime}} \delta_{i^{\prime}} b_{i^{\prime}}, \quad \chi_{p h}=\sum_{i^{\prime}} \zeta_{i^{\prime}} \phi_{i^{\prime}}, \quad \varphi_{p h}=\sum_{i^{\prime}} \eta_{i^{\prime}} \phi_{i^{\prime}}$ and $\chi_{p h}^{\prime}=\sum_{i^{\prime}} \theta_{i^{\prime}} \phi_{i^{\prime}}$. The additional fusion rules involving $b_{i^{\prime}}$ that we need are $b_{i^{\prime}} \times b_{j^{\prime}}=$ $\sum_{k^{\prime}} E_{i^{\prime} j^{\prime}}^{k^{\prime}} \phi_{k^{\prime}}, \quad b_{i^{\prime}} \times \psi_{j}=\sum_{k^{\prime}} D_{i^{\prime} j}^{k^{\prime}} \phi_{k^{\prime}}$

Previously, we discussed the constraint on the coefficients $\alpha_{i}, \beta_{j^{\prime}}$ originating from condition that the OPE of $\psi_{p h}$ with itself give $\phi_{p h}$ as a minimal dimension field. This requirement is an integral part of the conformal approach to solving the Hopf equations. In the present case we have similar conditions which must be imposed on the coefficients occuring in the definitions of $A_{p h}, . . \chi_{p h}^{\prime}$. Specifically we have

$$
\begin{aligned}
& A_{p h} \times A_{p h}=\varphi_{p h} \rightarrow \sum_{i^{\prime} j^{\prime}} \gamma_{i^{\prime}} \gamma_{j^{\prime}} E_{i^{\prime} j^{\prime}}^{k^{\prime}}=\eta_{k^{\prime}} \\
& A_{p h} \times \psi_{p h}=\chi_{p h} \rightarrow \sum_{i^{\prime} j} \gamma_{i^{\prime}} \alpha_{j} D_{i j}^{k^{\prime}}=\zeta_{k^{\prime}} \\
& B_{p h} \times \psi_{p h}=\chi_{p h}^{\prime} \rightarrow \sum_{i^{\prime} j} \delta_{i^{\prime}} \alpha_{j} D_{i^{\prime} j}^{k^{\prime}}=\theta_{k^{\prime}}
\end{aligned}
$$

So if we know $\alpha_{i}$ and $\gamma_{i^{\prime}}$ we can find the rest of the coefficients in the case of the 
parallel flow. The same holds for the perpendicular case if we know $\alpha_{i}$ and $\delta_{i^{\prime}}$. In fact we expect that a change in these parameters is related to changing the boundary conditions that leave the spectrum invariant and so represent a kind of universality. These are the free parameters of the solution.

At this stage we wish to make a few comments concerning solutions presented. It is apparent from Flohr's work [11] and the results presented here, that taking copies of a conformal model provides a way to incorporate the infrared condensate. If the copies are different then we have an asymmetry as can be seen from the following. Take as another possible solution $\tilde{c}_{6,1} \otimes c_{6,1}$. Then we have two $\Delta=-2$ and two $\Delta=-1$ fields, $\phi_{1}=\Psi_{(-1 \mid-1)}, \quad \phi_{2}=\Psi_{(-\tilde{1} \mid-1)}, \quad \psi_{1}=\Psi_{(-\tilde{1} \mid 0)}, \quad \psi_{2}=\Psi_{(\tilde{0} \mid-1)}$. However there is only one $\Delta=0$ field, namely $b=\Psi_{(1 \mid-1)}$. It follows that $b \times b=\phi_{1}$ and so this naturally provides another solution of the case of perpendicular flow only. There may be other asymmetric tensor product solutions of this type.

\section{Conclusions}

In this paper we have considered the possibility of describing solutions of $d=2$ ideal MHD turbulence within the framework of Flohr's logarithmic field theory solution in the pure fluid case. We have seen that the $\tilde{c}_{6,1} \otimes \tilde{c}_{6,1}$ theory has sufficient degeneracy of dimension $0,-1$ and -2 fields to be able to describe the additional fields one finds in the MHD case. Moreover the solutions of the corresponding Hopf equations was seen to be a consequence of the same hidden continuous symmetry that was exploited in the ordinary fluid case. This picture provides an interesting viewpoint of turbulent 2-d fluids and plasmas as corresponding to different regions of parameter space within a single model.

It would also be interesting to clarify further the exact relationship between the values of the real parameters entering the definitions of the physical fields $\psi_{p h}, \phi_{p h}, A_{p h}$ and $B_{p h}$ given earlier, and boundary conditions that would be appropriate in a given physical situation. This question was already touched upon in [11]. Also one can imagine that our results should be extendible to the case of "dyonic" or duality invariant MHD [20 ], whose minimal model conformal solutions have been investigated in [21].

Finally, there has been some interest in the past, in studying perturbations of CFT solutions to turbulence which break the conformal symmetry [22-24]. The application 
of such perturbations in LCFT's has been considered in [13] and [25] (and more recently in [27]). It would be interesting to apply these ideas to the particular MHD models we have considered here.

\section{Acknowledgments}

We would like to thank Oleg Soloviev for useful discussions. The work of SS was supported by the A. Onassis Public Benefit Foundation, and that of ST by the Royal Society.

\section{References}

[1] R.H. Kraichnan, 'Inertial ranges in two-dimensional turbulence', Phys. Fluids 10 (1967) 1417.

[2] A.M. Polyakov, Princeton Univ. Preprint PUPT-1341, hep-th/9209046.

[3] A.M. Polyakov Nucl. Phys. B396 (1993) 367.

[4] D.A. Lowe Mod. Phys. Lett. A8 (1993) 923.

[5] G. Falkovich and A. Hanany, 'Spectra of Conformal Turbulence ', Preprint WIS92-88-PH, hep-th/9212015 (1992).

[6] Y. Matsuo Mod. Phys. Lett. A8 (1993) 619.

[7] H. Cateau, Y. Matsuo and M. Umeski, 'Predictions on two-dimensional turbulence by conformal field theory', Preprint UT-652, hep-th/9310056 (1993).

[8] B.K. Chung, S. Nam, Q-H. Park and H.J. Shin, Phys. Lett. B309 (1993) 58.

[9] B.K. Chung, S. Nam, Q-H. Park and H.J. Shin, Phys. Lett. B317 (1993) 92.

[10] G.Falkovich, A.Hanany, Phys. Rev. Lett. 71 (1993) 3454-3457, hep-th/9301030

[11] M.A.I. Flohr Nucl.Phys. B482 (1996) 567-578, hep-th/9606130. 
[12] V. Gurarie, Nucl.Phys. B410 (1993) 535, hep-th/9303160

[13] J.S. Caux, I.I. Kogan, A.M. Tsvelik, Nucl.Phys. B466 (1996) 444.

[14] M.R.Rahimi Tabar, S. Rouhani, The Alf'ven Effect and Conformal Field Theory, hep-th/9507166; A Logarithmic Conformal Field Theory Solution For Two Dimensional Magnetohydrodynamics In Presence of The Alf'ven Effect, hepth/9606143.

[15] A. Shafiekhani, M.R.Tabar, Logarithmic Operators in Conformal Field Theory and the $W_{\infty}$, hep-th/9604007

[16] M. A.I. Flohr Int.J.Mod.Phys. A12 (1997) 1943-1958, hep-th/9605151

[17] L.C. Woods 'Principles of Magnetoplasma Dynamics ', Oxford Science publs. (1987).

[18] G. Ferretti and Z. Yang, Europhys. Lett. 22 (1993) 639.

[19] O. Coceal and S. Thomas, Mod. Phys. Lett.A10 (1995)

[20] O. Coceal, W.A. Sabra and S. Thomas, Europhys. Lett. 35 (5) (1996) 277.

[21] O. Coceal, W.A. Sabra and S.Thomas, Europhys. Lett. 35 (5) (1996) 343.

[22] O. Coceal and S.Thomas, Mod. Phys. Lett A10 (1995) 2427.

[23] Ph. Brax,A Renormalisation Group Analysis of 2d Freely Decaying Magnetohydrodynamic Turbulence, hep-th/9606156 The Coulomb Gas Behaviour of Two Dimensional Turbulence, hep-th/9505111

[24] L. Moriconi, 3-D Perturbations in Conformal Turbulence, hep-th/9508040

[25] I.I. Kogan and N.E. Mavromatos, Phys.Lett. B375 (1996) 111.

[26] I.I. Kogan and A. Lewis, Nucl.Phys. B509 (1998) 687.

[27] M.R. Rahimi Tabar, S. Rouhan Zamalodchikov's C-Theorem and The Logarithmic Conformal Field Theory, hep-th/9707060. 